\title{
Variação dos teores de constituintes voláteis de Cymbopogon citratus (DC) Staf, Poaceae, coletados em diferentes regiões do Estado de São Paulo
}

\author{
Marcos R. Furlan, ${ }^{1}$ Roberto Carlos C. Martins, ${ }^{2}$ Eliana Rodrigues, ${ }^{3}$ Nayara Scalco, ${ }^{3}$ \\ Giuseppina Negri, ${ }^{3}$ João Henrique G. Lago ${ }^{*, 4}$
}

'Departamento de Agronomia, Universidade de Taubaté, Rua Visconde do Rio Branco, 210, 12030-010 Taubaté-SP, Brasil,

${ }^{2}$ Centro Universitário Fundação Instituto Educacional de Osasco, Av. Franz Voegeli, 1743, 06020-190 Osasco-SP, Brasil,

${ }^{3}$ Departamento de Psicobiologia, Universidade Federal de São Paulo, Rua Botucatu, 862, 04023-900 São Paulo-SP, Brasil,

${ }^{4}$ Departamento de Ciências Exatas e da Terra, Universidade Federal de São Paulo, Rua Arthur Ridel, 275, 09972-270 Diadema-SP, Brasil.

\begin{abstract}
RESUMO: O óleo essencial extraído de Cymbopogon citratus (DC) Staf, Poaceae, é rico em citral sendo amplamente empregado na indústria química e farmacêutica. Os objetivos desse estudo foram avaliar o conteúdo de citral entre populações de $C$. citratus coletados em duas regiões diferentes (Ibiúna e Pindamonhangaba, Estado de São Paulo). O óleo essencial extraído de uma população cultivada em Pindamonhangaba-SP apresentou uma concentração alta de citral (aproximadamente $96,0 \%$ ), enquanto que o óleo extraído da população de Ibiúna-SP apresentou, além de citral, proporções elevadas de outro monoterpeno, o geraniol. Adicionalmente, os óleos essenciais obtidos desta população de C. citratus foram extraídos e analisados ao longo de $24 \mathrm{~h}$ (a cada $3 \mathrm{~h}$ ), sendo que o teor de citral foi maior no período da noite, o que mostra a importância de estudos que avaliam as diferenças químicas associadas às mudanças circadianas. Os componentes dos óleos essenciais foram identificados por $\mathrm{RMN}$ de ${ }^{13} \mathrm{C}, \mathrm{CG} / \mathrm{EM}$ e $\mathrm{CG}$ através do índice de Kovats.
\end{abstract}

Unitermos: Cymbopogon citratus, constituintes voláteis, citral.

\begin{abstract}
Variation in the amounts of volatile constituents of Cymbopogon citratus (DC) Staf, Poaceae, collected in different regions of São Paulo State." The essential oil extracted from Cymbopogon citratus (DC) Staf, Poaceae, is rich in citral and it has many applications in chemical and pharmaceutical industry. The aims of this study were to evaluate the amount of citral in populations of C. citratus collected in two different regions (Ibiúna and Pindamonhangaba, São Paulo State). The essential oil extracted of a cultivated population in Pidamonhangaba-SP, showed a high content of citral (approximately 96.0\%), while the essential oil from Ibiuna-SP, showed citral and high amounts of another monoterpene, geraniol. Additionally, the essential oils from this population of $C$. citratus were extracted and analyzed during $24 \mathrm{~h}$ (each $3 \mathrm{~h}$ ), being the major concentration of citral in the night period, indicating that studies which evaluated the chemical differences associated to circadian changes. The components of essential oils were identified through ${ }^{13} \mathrm{C}$ NMR, GC/MS, GC and Kovat's index.
\end{abstract}

Keywords: Cymbopogon citratus, volatile constituents, citral.

\section{INTRODUÇÃO}

Cymbopogon citratus (DC) Staf, Poaceae, é nativa das regiões tropicais da Ásia, cuja ocorrência vem sendo descrita nas Américas e na África (Souza \& Lorenzi, 2005). No Brasil é conhecida popularmente como capim- cidreira, erva-cidreira, capim-santo, capim-de-cheiro, citronela, capim-catinga, patchuli, capim-marinho, capim-membeca, palha-de-camelo, capim-ciri, capimcitronela e esquenanto (Lorenzi \& Matos, 2002). É uma planta aromática cultivada para a produção comercial de 
óleo essencial, o qual também é utilizado como material de partida para síntese da vitamina A (Vieira, 2006).

O consumo da planta pela população se dá essencialmente pela forma de chá (Craveiro et al., 1981; Lorenzi \& Matos, 2002), o qual deve ser preparado de preferência com folhas frescas. Do ponto de vista etnofarmacológico, as folhas são utilizadas na forma de infusão para ingestão nos casos de disenteria, problemas digestivos, febre, dor de cabeça, fraqueza dos nervos, agitação do sangue, gripe, pequenas crises de cólica uterina e intestinal, insônia, intranqüilidade; como calmante, sedativa nas doenças nervosas, sudorífera, carminativa, diurética e anti-reumática (Carrara, 1995; Almeida, 2003; Silva \& Andrade, 2004; Albuquerque, 2006; Freitas \& Rodrigues, 2006; Vieira, 2006). Na Amazônia, o chá das folhas é também utilizado como calmante e analgésico em casos de cefaléia além de agir como antigripal, hipotensor, estimulante do aparelho digestivo e diurético enquanto que a decocção é usada contra febre, males da garganta e sintomas gripais em geral (Santos, 1994; Schuck et al., 2001). As raízes são utilizadas em decoctos, para facilitar o trabalho de parto (Rodrigues, 2006). No pantanal, as raízes, caules e folhas são utilizados na forma de chá como expectorante, calmante, para o combate da pressão alta, dor, gripe, reumatismo, febre, problemas renais, gases intestinais, estresse, distúrbios do sono e para problemas do coração, limpeza uterina pós-parto e no combate a gripe e tosse (Assunção et al., 1993; De La Cruz, 1997; Silva, 1997; Sulzbach, 1998; Faria, 1998; Rodrigues \& Carlini, 2004).

Dentre as principais substâncias estão os derivados encontrados nos óleos essenciais, cuja composição química pode variar sendo influenciada pela diversidade genética e o habitat (Santos et al., 2009). Análises do óleo essencial de várias regiões identificaram o citral (mistura de neral e geranial) como o principal constituinte (Andrade et al., 2009). A análise de doze amostras brasileiras realizadas por $\mathrm{CG} / \mathrm{EM}$ indicaram a presença de 22 substâncias, sendo neral e geranial os principais constituintes com variações de 40,7 a 75,4\% (Barbosa et al., 2008). No entanto, no óleo essencial de C. citratus originário da Etiópia, geraniol foi encontrado como principal substância, correspondendo a $40 \%$ do óleo (Abegaz \& Yohannes, 1983). No originário de Marrocos, os principais constituintes encontrados foram geranial (39,8\%) e neral (32\%) (Il Idrissi et al., 1993), enquanto que naqueles originários da Zâmbia e do Zimbabue foram encontrados geranial, neral e mirceno em diferentes proporções porém com expressiva predominância dos dois primeiros (Chisowa et al., 1998; Chagonda et al., 2000). No óleo essencial de uma amostra da Nigéria foram encontrados geranial (40,9\%), neral (29,7\%), mirceno $(11,3 \%)$, linalool $(1,7 \%)$ e acetato de geranila (1,6\%) (Owolabi et al., 2008).

Dada a importância farmacológica e comercial do óleo essencial de C. citratus (Negrelle \& Gomes, 2007; Santos et al., 2009), neste trabalho, o óleo essencial proveniente de uma população oriunda de Pindamonhangaba-SP foi obtido através de hidrodestilação e os teores de neral e geranial foram determinados através de CG e CG/EM. No entanto, apesar de haver a descrição dos componentes voláteis de espécimes de $C$. citratus analisados em diferentes regiões, poucos estudos foram realizados visando avaliar possíveis variações no teor dos principais constituintes dos óleos essenciais. Desta forma, visando-se verificar a influência de fatores geográficos e microclimáticos na composição química dos óleos essenciais, foi selecionada outra população de C. citratus, oriunda de Ibiúna-SP. Adicionalmente, foram realizadas diferentes coletas ao longo de um mesmo dia com essa população, visando-se observar ocorrência de variação circadiana. Através de análise por CG, CG/EM e RMN, a proporção relativa dos principais constituintes dos óleos essenciais foram também determinadas, visando detectar variações nas proporções desses principais constituintes.

\section{MATERIAL E MÉTODOS}

\section{Material vegetal}

No município de Pindamonhangaba-SP (232'34'S e 45'31'02'O, com altitude média de $577 \mathrm{~m}$ ), foi realizada uma coleta de folhas frescas de Cymbopogon citratus (DC) Staf, Poaceae,, (aproximadamente $600 \mathrm{~g}$ ) a partir de uma população cultivada no pólo Regional de Desenvolvimento Tecnológico dos Agronegócios do Vale do Paraíba. No município de Ibiúna (23³9'23"S e $47^{\circ} 13^{\prime} 21^{\prime \prime} \mathrm{O}$, altitude média de $850 \mathrm{~m}$ ) foram realizadas oito coletas de folhas de uma mesma população (aproximadamente $200 \mathrm{~g}$ ), as quais foram iniciadas às $12 \mathrm{~h} 30 \mathrm{~min}$ e repetidas em períodos de $3 \mathrm{~h}$ a partir deste horário ao longo de $24 \mathrm{~h}$. Em ambos os municípios, as coletas foram realizadas entre os dias 10 e 11 de janeiro de 2008. A identificação da espécie estudada foi realizada pelo Prof. João Carlos Nordi do Centro de Estudos de Plantas Medicinais do Departamento de Ciências Agrárias da Unitau, onde uma exsicata (número 59) encontra-se depositada. Os tipos de solos foram caracterizados como latossolo vermelho amarelado distrófico com textura franco argiloso-arenoso (Palmieri et al., 2003).

\section{Obtenção dos dados microclimáticos}

Os dados microclimáticos de temperatura e de precipitação em cada região de coleta foram registrados, respectivamente, com um termo-anemômetro Pocket Weather Meter Kestrel 3000 (Nielsen-Kellerman-USA) e um pluviômetro construído em nosso laboratório (valores em milímetros). Os dados obtidos foram comparados 
com aqueles fornecidos pelo Instituto Nacional de Pesquisas Espaciais, a partir de estações localizadas nas proximidades das regiões de coleta.

\section{Extração dos óleos essenciais}

A cada período de coleta, o óleo essencia das folhas foi extraído utilizando-se um aparelho de Clevenger adaptado a um balão de fundo redondo com capacidade de $1000 \mathrm{~mL}$. As folhas frescas das amostras coletadas em Ibiúna-SP e das amostras coletadas em Pindamonhangaba-SP foram cortadas em frações de aproximadamente $2 \mathrm{~cm}$, colocadas no balão e recobertas com água destilada (aproximadamente $500 \mathrm{~mL}$ ). O processo de destilação foi realizado em um período de 2,5 h, mantendo-se a solução em ebulição. O óleo bruto foi retirado com o auxílio de uma pipeta de Pasteur, secado com $\mathrm{Na}_{2} \mathrm{SO}_{4}$ anidro, filtrado, acondicionado em frasco de vidro âmbar e armazenados em freezer $\left(-20^{\circ} \mathrm{C}\right)$. Os rendimentos das extrações foram calculados com base na massa dos óleos brutos obtidos em relação à massa de material vegetal fresco (Lago et al., 2002; Lago \& Roque, 2002; Lago et al., 2006).

\section{Análise por CG e CG/EM}

A análise por CG foi realizada em um cromatógrafo Hewlett-Packard 5890, séries II (gás de arraste: $\mathrm{He}$ ) equipado com detector FID, coluna capilar HP-5 (5\% de difenil em 95\% de dimetilpolisiloxano, comprimento: $30 \mathrm{~m}$, diâmetro interno: $0,32 \mathrm{~mm}$, espessura do filme: $0,25 \mu \mathrm{m})$, injetor automático (HP 7673 ) e integrador eletrônico (HP 3396A). Programação de temperatura do forno: $60-280{ }^{\circ} \mathrm{C}$ com taxa de aquecimento de $3{ }^{\circ} \mathrm{C} / \mathrm{min}$ mantendo-se $10 \mathrm{~min}$ a $280^{\circ} \mathrm{C}$. As temperaturas do injetor e do detector foram mantidas em $220^{\circ} \mathrm{C}$ e $280^{\circ} \mathrm{C}$, respectivamente. As concentrações dos componentes foram calculadas tendo como base as áreas dos picos no cromatograma, seguindo a ordem de eluição da coluna HP-5, analisados em triplicata. A análise por $\mathrm{CG} / \mathrm{EM}$ foi realizada em cromatógrafo a gás Shimadzu, modelo CG-17A, acoplado com espectrômetro de massas MS-QP-5050A, operando por impacto eletrônico $(70 \mathrm{eV})$. Foi utilizada uma coluna DB-5 com $30 \mathrm{~m}$ de comprimento e $0,25 \mathrm{~mm}$ de diâmetro interno e hélio como gás de arraste ( $1 \mathrm{~mL} / \mathrm{min})$, nas mesmas condições de programações de temperatura descritas acima.

\section{Análise por ressonância magnética nuclear (RMN)}

Os espectros de RMN foram registrados em um espectrômetro Bruker, modelo DPX-300, operando a $75 \mathrm{MHz}$ para o núcleo do carbono-13, sendo utilizado clorofórmio-d (Aldrich) como solvente e o pico residual do derivado não deuterado empregado como padrão interno (Brochini \& Lago, 2007).

\section{Identificação dos constituintes}

Para identificação dos componentes do óleo essencial foi realizada a comparação dos espectros de massas utilizando o programa CLASS-5000, versão 2.23 da Shimadzu Corporation, equipado com as bibliotecas NIST 21, NIST 107 e WILEY 229. A confirmação estrutural foi efetuada através do cálculo do índice de Kovats para cada componente, determinado relativamente aos tempos de retenção de uma série de n-alcanos (Adams, 2001; Lago et al., 2006).

\section{RESULTADOS E DISCUSSÃO}

Os rendimentos dos óleos essenciais das folhas das diferentes coletas de Cymbopogon citratus (DC) Staf, Poaceae, estão mostrados na Tabela 1. Desta forma, do óleo obtido em Pindamonhangaba-SP, o rendimento foi de 2,5\% enquanto que para os óleos provenientes de Ibiúna-SP, os mesmos foram determinados como $2,4 \pm 0,1 \%$ em todas as coletas, não sendo observada, desta maneira, variação significativa, apesar dos parâmetros microclimáticos de temperatura e precipitação apresentarem expressiva diferenciação.

Os componentes majoritários dos óleos essenciais de $C$. citratus foram identificados como neral (1) e geranial (2) através de CG/EM, cujos teores foram quantificados pela análise do cromatograma obtido por CG. A ocorrência dos dois isômeros pode ser comprovada através da análise do espectro de $\mathrm{RMN}$ de ${ }^{13} \mathrm{C}$ do óleo essencial bruto (Brochini et al., 1999; Brochini \& Lago, 2007), o qual mostrou, dentre outros, sinais relativos a dois carbonos carbonílicos em $\delta 190,9$ e em $\delta 191,4$, os quais puderam ser atribuídos aos carbonos $\mathrm{C}-1$ do neral e do geranial, respectivamente. Além destes, outros sinais foram detectados entre $\delta$ 164,0-122,4, relativos aos carbonos $\mathrm{sp}^{2} \mathrm{C}-2 / \mathrm{C}-3$ e C-6/C-7 da mistura isomérica. Através da comparação dos dados obtidos com aqueles descritos na literatura (Feger et al., 2006; Pihlasalo et al., 2007), foi possível identificar os dois monoterpenos na mistura.

Como podem ser observado na Tabela 2, no óleo essencial proveniente de Pindamonhangaba-SP, os teores dos monoterpenos neral e geranial foram $42,1 \pm 0,2$ e $56,1 \pm 0,1 \%$, respectivamente. Por outro lado, no óleo obtido de Ibiúna-SP, os teores dos mesmos monoterpenos foram inferiores, sendo determinados como $18,5 \pm 0,6$ e $22,2 \pm 0,6 \%$, respectivamente. No entanto, não se observou somente uma diminuição no teor de citral, mas principalmente a detecção de teor elevado de outro monoterpeno, o geraniol (3) $(28,56 \pm 0,01 \%)$, cuja alteração pode ter sido causada por fatores fitogeográficos e microclimáticos (Gobbo-Netto \& Lopes, 2007), principalmente pluviosidade, visto 
que em Pindamonhangaba-SP foram observados 1310 $\mathrm{mm}$ de chuva no mês de coleta ao passo que não houve precipitação no mês de janeiro em Ibiúna-SP.

Visando-se detectar variação nas proporções relativas de monoterpenos dos óleos provenientes das folhas devido a variações circadianas, esse estudo foi direcionado para a avaliação dos teores dos óleos essenciais de $C$. citratus obtidos em Ibiúna-SP, em oito coletas realizadas ao longo de $24 \mathrm{~h}$. De acordo com o observado nas Tabelas 2 e 3, o teor de citral obtido em todas as coletas realizadas em Ibiúna-SP foi inferior ao detectado em Pindamonhangaba-SP, uma vez que o teor máximo da mistura desses dois derivados isoméricos foi de $49 \%$. Foi observado que o teor de citral sofreu pouca variação ao longo do dia, sendo maior apenas no período da noite, ou seja, as $18 \mathrm{~h} 30(49,3 \pm 0,4 \%)$ e as $21 \mathrm{~h} 30(46,97 \pm 0,07 \%)$, período onde se observou uma diminuição nos valores de temperatura (Tabela 1), sugerindo a ação desse fator na produção e acúmulo destes metabólitos (Gobbo-Netto \& Lopes, 2007). No começo da madrugada a proporção desses derivados sofreu nova diminuição sendo detectado um teor de $42,1 \pm 0,1 \%$ às $00 \mathrm{~h} 30$, o qual permaneceu até o meio da manhã $(9 h 30,40,7 \pm 0,6 \%)$. No entanto, se o teor de neral + geranial diminuiu no início da noite, a proporção relativa de geraniol passou de $30,1 \pm 0,1 \%$ às $15 \mathrm{~h} 30$ para $32,7 \pm 0,2 \%$ às $18 \mathrm{~h} 30$. Do mesmo modo, o teor de geraniol foi maior às $21 \mathrm{~h} 30(33,7 \pm 0,2 \%)$, diminuindo novamente para a faixa dos $29 \%$ às $00 \mathrm{~h} 30 \mathrm{e}$ assim permanecendo até o meio do dia.

Tabela 1. Rendimento dos óleos essenciais e valores de temperatura e precipitação nos períodos de coleta de Cymbopogon citratus (DC) Staf, Poaceae,em Pindamonhangaba-SP e Ibiúna-SP.

\begin{tabular}{lccccccccc}
\hline & \multicolumn{7}{c}{ Ibiúna-SP } \\
& \multirow{2}{*}{ Pindamonhangaba-SP } & $12 h 30$ & $15 h 30$ & $18 h 30$ & $21 h 30$ & $0 h 30$ & $3 h 30$ & $6 h 30$ & $9 h 30$ \\
\hline Rendimento/\% & 2,5 & 2,5 & 2,5 & 2,4 & 2,4 & 2,3 & 2,4 & 2,4 & 2,5 \\
Precipitação*/mm & 1310 & 0 & 0 & 0 & 0 & 0 & 0 & 0 & 0 \\
Temperatura/ $/{ }^{\circ} \mathrm{C}$ & 21 & 27 & 25 & 18 & 14 & 13 & 15 & 20 & 22 \\
\hline
\end{tabular}

*dados do mês de janeiro/2008

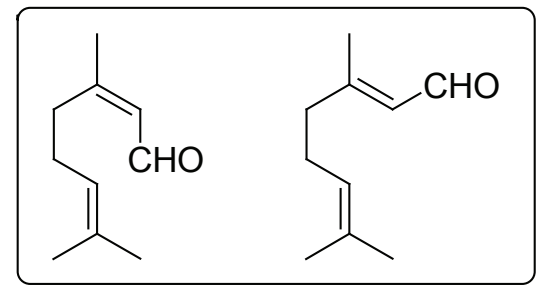

1

2

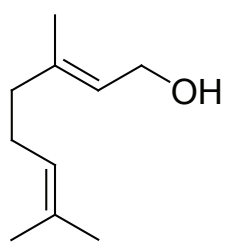

3

Tabela 2. Proporção relativa dos principais derivados identificados no óleo essencial de Cymbopogon citratus (DC) Staf, Poaceae, proveniente de espécimes oriundos de Pindamonhangaba-SP e de Ibiúna-SP.

\begin{tabular}{lcc}
\hline \multirow{2}{*}{ Componente } & \multicolumn{2}{c}{ Local de coleta } \\
\cline { 2 - 3 } & Pindamonhangaba-SP & Ibiúna-SP \\
\hline Neral & $42,1 \pm 0,2$ & $18,5 \pm 0,6$ \\
Geranial & $56,1 \pm 0,1$ & $22,2 \pm 0,6$ \\
Geraniol & - & $28,56 \pm 0,01$ \\
\hline Total & $98,2 \pm 0,2$ & $69,3 \pm 0,6$ \\
\hline
\end{tabular}

Tabela 3. Proporção relativa dos principais derivados identificados no óleo essencial de Cymbopogon citratus (DC) Staf, Poaceae, proveniente de Ibiúna-SP, em coletas realizadas ao longo de um dia, a cada 3 horas.*

\begin{tabular}{|c|c|c|c|c|}
\hline Horário & neral & geranial & geraniol & Total \\
\hline $12 \mathrm{~h} 30$ & $19,3 \pm 0,1$ & $23,2 \pm 0,1$ & $30,1 \pm 0,2$ & $72,6 \pm 0,2$ \\
\hline $15 \mathrm{~h} 30$ & $19,5 \pm 0,2$ & $23,3 \pm 0,2$ & $30,1 \pm 0,1$ & $72,9 \pm 0,2$ \\
\hline $18 \mathrm{~h} 30$ & $22,4 \pm 0,4$ & $26,9 \pm 0,4$ & $32,7 \pm 0,2$ & $82,0 \pm 0,4$ \\
\hline $21 \mathrm{~h} 30$ & $21,4 \pm 0,07$ & $25,6 \pm 0,07$ & $33,7 \pm 0,2$ & $80,7 \pm 0,2$ \\
\hline $00 \mathrm{~h} 30$ & $19,1 \pm 0,1$ & $23,0 \pm 0,1$ & $29,5 \pm 0,09$ & $71,6 \pm 0,1$ \\
\hline $03 \mathrm{~h} 30$ & $18,8 \pm 0,7$ & $22,6 \pm 0,7$ & $29,8 \pm 0,3$ & $71,2 \pm 0,7$ \\
\hline $06 h 30$ & $18,9 \pm 0,1$ & $22,7 \pm 0,1$ & $28,5 \pm 0,1$ & $70,1 \pm 0,1$ \\
\hline $09 \mathrm{~h} 30$ & $18,5 \pm 0,6$ & $22,2 \pm 0,6$ & $28,6 \pm 0,01$ & $69,3 \pm 0,6$ \\
\hline \multicolumn{5}{|c|}{ * análises realizadas em triplicata } \\
\hline & & & & $\begin{array}{l}\text { Rev. Bras. Farmacogn. } \\
\text { Braz. J. Pharmacogn. } \\
\text { 20(5): Out./Nov. } 2010\end{array}$ \\
\hline
\end{tabular}




\section{CONCLUSÃO}

Tendo em vista que muitas das atividades biológicas observadas para $C$. citratus são atribuídas ao citral, o qual é frequentemente obtido em altas concentrações nesta espécie, pôde-se detectar, como resultados deste trabalho, que o teor desses monoterpenos pode variar devido a ocorrências de fatores fitogeográficos e/ou microclimáticos. Como observado, na espécie proveniente de Ibiúna-SP, apesar de não apresentar variação nos teores dos monoterpenos ao longo de 24 $\mathrm{h}$, os teores de citral foram menores do que aqueles detectados nos óleos de Pindamonhangaba-SP, com a expressiva ocorrência de geraniol, um derivado reduzido do geranial. Desta forma, os dados obtidos indicam a importância do local e horário de coleta de material, principalmente quando o mesmo é utilizado para fins medicinais.

\section{AGRADECIMENTOS}

Os autores agradecem as agencias de fomento (FAPESP e CNPq) pelo apoio financeiro para o desenvolvimento dos projetos e as bolsas concedidas.

\section{REFERÊNCIAS}

Abegaz B, Yohannes PG 1983. Constituents of the essential oil of Ethiopian Cymbopogon citratus Stapf. J Nat Prod 46: 424-426.

Adams RP 2001. Identification of Essential Oil Components by Gas Chromatography/Quadrupole Mass Spectroscopy. Carol Stream: Allured Publ. Corp.

Albuquerque UP 2006. Re-examining hypotheses concerning the use and knowledge of medicinal plants: a study in the caatinga vegetation of NE Brazil. J Ethnobiol Ethnomed 26: 2-30.

Almeida MZ 2003. Plantas Medicinais. Salvador: EDUFBA.

Andrade EHA, Zoghbi MGB, Lima MD 2009. Chemical composition of the essential oils of Cymbopogon citratus (DC.) Stapf cultivated in North of Brazil. $J$ Essent Oil Bearing Plants 12: 41-45.

Assunção L, Paulino LM, Leão MG 1993. Utilização popular de plantas medicinais no município de Cáceres, Estado de Mato Grosso. Trabalho de Especialização. Instituto de Saúde Coletiva da Universidade Federal do Mato Grosso.

Barbosa LCA, Pereira UA, Martinazzo AP, Maltha CRA, Teixeira RR, Melo EC 2008. Evaluation of the chemical composition of Brazilian commercial Cymbopogon citratus (DC) Stapf samples. Molecules 13: 1864-1874.

Brochini CB, Lago JHG 2007. Aplicação de técnicas cromatográficas e espectrométricas como ferramentas de auxílio na identificação de componentes de óleos voláteis. Rev Bras Farmacogn 17: 266-270.

Brochini CB, Núñez CV, Moreira IC, Roque NF, Chaves MH,
Martins D 1999. Identificação de componentes de óleos voláteis: análise espectroscópica de misturas de sesquiterpenos. Quim Nova 22: 37-40.

Carrara D 1995. Possangaba: o pensamento médico popular. Marica: Ribro Soft.

Chagonda LS, Makanda C, Chalchat JC 2000. Essential oils of cultivated Cymbopogon winterianus (Jowitt) and of $C$. citratus (DC) (Stapf) from Zimbabwe. J Essent Oil Res 12: 478-480.

Chisowa EH, Hall DR, Farman DI 1998. Volatile constituents of the essential oil of Cymbopogon citratus Stapf grown in Zambia. Flavour Fragr J 13: 29-30.

Craveiro AA, Fernandez AG, Andrade CHS 1981. Óleos essenciais de plantas do Nordeste. Fortaleza: Editora da UFC.

De La Cruz MGF 1997. Plantas medicinais utilizadas por raizeiros: Uma abordagem etnobotânica no contexto da saúde e doença. Cuiabá, MT. Cuiabá, 251p. Dissertação de mestrado. Universidade Federal do Mato Grosso.

Faria APOC 1998. O uso de plantas medicinais em Juscimeira e Rondonópolis, Mato Grosso: Um estudo etnoecológico. Cuiabá, 187p. Dissertação de mestrado. Instituto de Biociências. Universidade Federal do Mato Grosso.

Feger W, Brandauer H, Gabris P, Ziegler H 2006. Nonvolatiles of commercial lime and grapefruit oils separated by high-speed countercurrent chromatography. J Agric Food Chem 54: 2242-2252.

Freitas TA, Rodrigues ACC 2006. Etnobotânica das plantas medicinais de Madre Deus, Bahia. Sitientibus Série Biológicas 6: 133-137.

Gobbo-Netto L, Lopes NP 2007. Medicinal plants: Factors of influence on the content of secondary metabolites. Quim Nova 30: 374-381.

Il Idrissi A, Bellakhdar J, Canigueral S, Iglesias J, Vila R 1993. Composition of the essential oil from citronnelle (Cymbopogon citratus (DC.) Stapf) acclimatized in Morocco. Plant Med Phytother 26: 274-277.

Lago JHG, Reis AA, Roque NF 2002. Chemical composition from volatile oil of the stem bark of Guarea macrophylla Vahl. ssp. tuberculata Vellozo (Meliaceae). Flavour Frag J 17: 255-257.

Lago JHG, Roque NF 2002. Terpenes from the essential oil of the leaves of Guarea macrophylla Vahl. ssp. tuberculata Vellozo (Meliaceae). J Essent Oil Res 14: 12-13.

Lago JHG, Soares MG, Batista-Pereira LG, Silva MFGF, Corrêa AG, Fernandes JB, Vieira PC, Roque NF 2006. Volatile oil from Guarea macrophylla ssp. tuberculata: seasonal variation and electroantennographic detection by Hypsipyla grandella. Phytochemistry 67: 589-594.

Lorenzi H, Matos FJA 2002. Plantas medicinais no Brasil: nativas e exóticas. Nova Odessa: Plantarum.

Negrelle RRB, Gomes EC 2007. Cymbopogon citratus (DC) Stapf: chemical and pharmacological review. Rev Bras Pl Med 9: 80-92.

Owolabi MS, Oladimeji MO, Lajide L, Singh G, Marimuthu P, Isidorov VA 2008. Cymbopogon citratus (DC) Stapf 
volatile oil from South-West, Nigeria. J Essent Oil Bearing Plants 11: 335-341.

Palmieri F, Santos HG, Gomes IA, Lumbreras JF, Aglio MLD 2003. The Brazilian soil classification system. In: Eswaran H, Rice T, Ahrens R, Stewart BA (Ed.). Soil classification: a global desk reference. Boca Raton: CRC Press, 127-146.

Pihlasalo J, Klika KD, Murzin DY, Nieminen V 2007. Conformational equilibria of citral. $J$ Molecular Structure-Theochem 814: 33-41.

Rodrigues E, Carlini EA 2004. Plants used by a quilombola group in Brazil with potential central nervous system effects. Phytother Res 18: 748-753.

Rodrigues E 2006. Plants and animals utilized as medicines in the Jaú National Park (JNP), Brazilian Amazon. Phytother Res 20: 378-391.

Santos A, Paduan RH, Gazin ZC, Jacomassi E, D'Oliveira PS, Cortez DAG, Cortez LER 2009. Determinação do rendimento e atividade antimicrobiana do óleo essencial de Cymbopogon citratus (DC.) Stapf em função de sazonalidade e consorcimento. Rev Bras Farmacogn 19: 436-441.

Santos PD 1994. Formação de um banco de dados sobre plantas medicinais da Amazônia. Programa Interinstitucional de Iniciação Científica. INPA/CPPN,

Schuck VJA, Fratini M, Rauber CS, Henriques A, Schapoval EES 2001. Avaliação da atividade antimicrobiana de Cymbopogon citratus. Rev Bras Cien Farm 37: 45-49.

Silva MAP 1997. Meio ambiente, educação e flora local: Saber e fazer em Nossa Senhora da Guia, MT. Cuiabá, 171p. Dissertação de mestrado, Instituto de Educação da Universidade Federal do Mato Grosso.

Silva VA, Andrade LHC 2004. O significado cultural das espécies botânicas entre indígenas de Pernambuco: o caso Xucuru. Biotemas 7: 79-94.

Souza VC, Lorenzi H 2005. Botânica sistemática: guia ilustrado para identificação das famílias de Angiospermas da flora brasileira, baseado em APG II. Nova Odessa: Plantarum.

Sulzbach AW 1998. O uso de plantas medicinais no Bairro Pascoal Ramos (Cuiabá, MT). Cuiabá. Trabalho de especialização. Instituto de Biociências da Universidade Federal do Mato Grosso.

Vieira AV 2006. Influência do espaçamento, altura de corte e idade de primeiro corte na produtividade de capimsanto. Fortaleza, 38p. Dissertação de mestrado. Universidade Federal do Ceará. 